\title{
Relationship between Mandibular Position and Oropharyngeal Space in Obstructive Sleep Apnea Syndrome Patients with Dental Oral Appliance
}

\author{
Yoshimi Inoko, DDS, PhD, and Osami Morita, DDS, PhD \\ Department of Complete Denture, The Nippon Dental University School of Life Dentistry at Niigata, Niigata, Japan
}

\section{Clinical significance}

The results of this study showed that a dental oral appliance (OA) designed to advance the mandible forward is a useful cure for obstructive sleep apnea syndrome (OSAS) and that excessive increase in the vertical dimension with the OA is of no value in increasing the oropharyngeal space.

\begin{abstract}
Purpose: The purpose of this study was to verify the usefulness of an oral appliance (OA) in patients with obstructive sleep apnea syndrome (OSAS), and to examine the relationship between the shift in mandibular position and fluctuations in the oropharyngeal space.

Methods: Fourteen patients ( 5 females and 9 males; mean age 51.1 years) diagnosed with OSAS were evaluated by pulse oximetry before and after insertion of the OA. Similarly, lateral cephalometric radiographs for each patient were obtained while sitting and awake in an upright position, in the intercuspal position, with the OA and at maximum opening.

Results: Using the OA, the oropharyngeal space increased significantly with the exception of the top of oropharynx. At maximum opening, the space significantly decreased in the lower part of the oropharynx, however the space in the upper part of the oropharynx was significantly increased. In the lower part of oropharynx using OA, there was a relationship between the anterior shift in mandibular position and the increase in oropharyngeal space. However, there was no significant relationship between the downward shift in mandibular position and the increase in oropharyngeal space. The oxygen desaturation index and cumulative percentage of time spent at saturations below $90 \%$ during sleeping were significantly decreased in patients using OA.
\end{abstract}

\section{Corresponding to: Dr Yoshimi Inoko}

Department of Complete Denture, The Nippon Dental

University School of Life Dentistry at Niigata

1-8 Hamaura-cho, Chuo-ku, Niigata 951-8580, Japan

Tel: +81-25-267-1500, Fax: +81-25-267-8906

E-mail: yinoko@ngt.ndu.ac.jp

Received on December 15, 2006 / Accepted on June 4, 2007
Conclusion: These results confirm that the OA is useful as a cure for patients with OSAS and it suggests that the excessive increase in occlusal vertical dimension using the OA is of no value in expanding the oropharyngeal space.

Key words: obstructive sleep apnea syndrome, dental oral appliance, lateral cephalogram, oropharyngeal space, pulse oximetry

\section{Introduction}

Sleep apnea is probably the most common of all the sleep disorders and obstructive sleep apnea syndrome (OSAS) is the most prevalent of the sleep apneas. OSAS is caused by partial or complete obstruction of the upper airway during sleep. Polysomnographic evaluation is required for the definitive diagnosis of sleep disorders such as OSAS. Without adequate ventilation, the blood carbon dioxide pressure eventually increases to a certain level that awakens the patient. Consequently, the patient awakens briefly, inhales, and then returns to sleep without consciously remembering the episode. ${ }^{1,2}$ The principal effects of OSAS are loud and irregular snoring, daytime fatigue, and sleepiness, which are caused by sleep fragmentation. Therefore, patients with this condition are at increased risk of death, for example, from automobile accidents. ${ }^{3}$ Conservative treatments of OSAS included weightlessness, changes in sleep position, medicine therapy, nasal continuous positive airway pressure (NCPAP) and placement of an intraoral prosthesis. Although NCPAP is the most common treatment method for OSAS, ${ }^{4}$ it is often too demanding to be accepted by many patients. Uvulopalatopharyngoplasty, maxillomandibular surgery and dental oral appliances (OA) have been proposed as alternatives to NCPAP. ${ }^{5}$ In recent years, OAs that modify the position of the mandible have been advocated as treatment 
Table 1 Clinical characteristics of the subjects.

\begin{tabular}{lccccc}
\hline & & average & median & s.d. & range \\
\hline Age & $($ Years $)$ & 51.0 & 51.5 & 12.8 & $31-70$ \\
Body mass index & $\left(\mathrm{kg} / \mathrm{m}^{2}\right)$ & 23.7 & 24.6 & 2.7 & $16-26.1$ \\
Apnea hypopnea index $(/ \mathrm{h})$ & 18.1 & 18.0 & 9.2 & $6.7-37.0$ \\
\hline
\end{tabular}

in patients with mild OSAS. Various OAs are used frequently for the treatment of temporomandibular disorders, nocturnal bruxism and more recently for the treatment of sleep apnea or snoring. Several researchers have indicated that the ability to use OAs for OSAS has significantly improved efficiency of the treatment. ${ }^{6,7}$ Namely, the OA increases the oropharyngeal airway space allowing more air to pass through the space during inspiration.

Lowe AA et $\mathrm{al}^{8}$ reported that OAs had a success rate of up to $80 \%$ in mild cases of OSAS and $60 \%$ in more severe cases. Many imaging studies ${ }^{9-12}$ have shown that OAs advance the mandible forward and increase the space within the upper airway. These results may be related to a dose-dependent enlargement of the upper airway, ${ }^{13}$ augmentation of the upper airway muscle active $^{14}$ and improvement of minimum nocturnal arterial oxygen saturation because of mandibular protrusion. In the clinic, OSAS decreases as the mandible is advanced with an OA, and each patient has a comfortable forward mandibular position in which reduction or cessation of OSAS is reported. However, the relationship between the condyle position and the acceptable range of mandibular advancement with OAs in patients with mild OSAS is not well investigated.

The purposes of this study were to evaluate the relationship between different amounts of mandibular protrusion with an OA and the horizontal extent of the oropharyngeal space after the insertion of the OA and in the maximum opening position, and to define the effect of an OA on percutaneous arterial oxygen saturation $\left(\mathrm{SpO}_{2}\right)$ in patients with OSAS.

\section{Materials and methods}

\section{Subjects}

Fourteen patients ( 5 females and 9 males) with OSAS participated in this study (Table 1). They were accepted into the study if they had more than 8 teeth in each jaw, ${ }^{15}$ had no symptoms re- lated to temporomandibular disorders and had a body mass index (BMI) less than $33 \mathrm{~kg} / \mathrm{m}^{2}{ }^{2}{ }^{16} \mathrm{~A}$ physician screened patients in The Nippon Dental University Niigata Hospital. They were referred to The Nippon Dental University Niigata Hospital because of symptoms compatible with snoring. The diagnosis of obstructive sleep apnea was based on polysomnographic findings. The study was performed with the approval of the Ethics Committee of The Nippon Dental University School of Life Dentistry at Niigata (\#42), and informed consent was obtained from all patients.

\section{Appliance description}

After each patient had undergone a baseline medical, dental, and stomatognathic examination, an OA was fabricated. The OA was fabricated individually for each patient using the method of Harada et al. ${ }^{17}$

After checking for retentiveness of the maxillary and mandibular splints, the degree of protrusion of the OA was adjusted for individual patients. The splints were attached together in the patient's mouth with a self-curing resin. The patients were instructed to only use the OA during sleep and were also informed of the possible side effects, such as transient tooth, temporomandibular joint, or masticatory muscle discomfort that may occur the following morning after initial use. If the patient had marked discomfort at the temporomandibular joint or masticatory muscle, it was possible to separate and rejoin the device at a decreased protrusive position. The adjustments were repeated until discomforts disappeared.

\section{Cephalometric evaluation}

Three lateral cephalometric radiographs for each patient were obtained with the patient sitting and awake in an upright position with the Frankfort horizontal plane parallel to the floor. For the first radiograph, the patient was instructed to make light contact in the intercuspal position; for the second radiograph, the patient was instructed to adopt the maximum opening position; and for the third radiograph, the OA was fitted intraorally. No instructions regarding tongue position were given to the patients. All lateral skull radiographs were taken with a cephalometric radiograph unit (Asahi Roentgen Ind. Co. LTD, CX-150SK) using a standard technique. The distance from the focus to the median plane was $1.5 \mathrm{~m}$, and enlargement was $11 \%$, which was not corrected in the cephalometric analysis. 


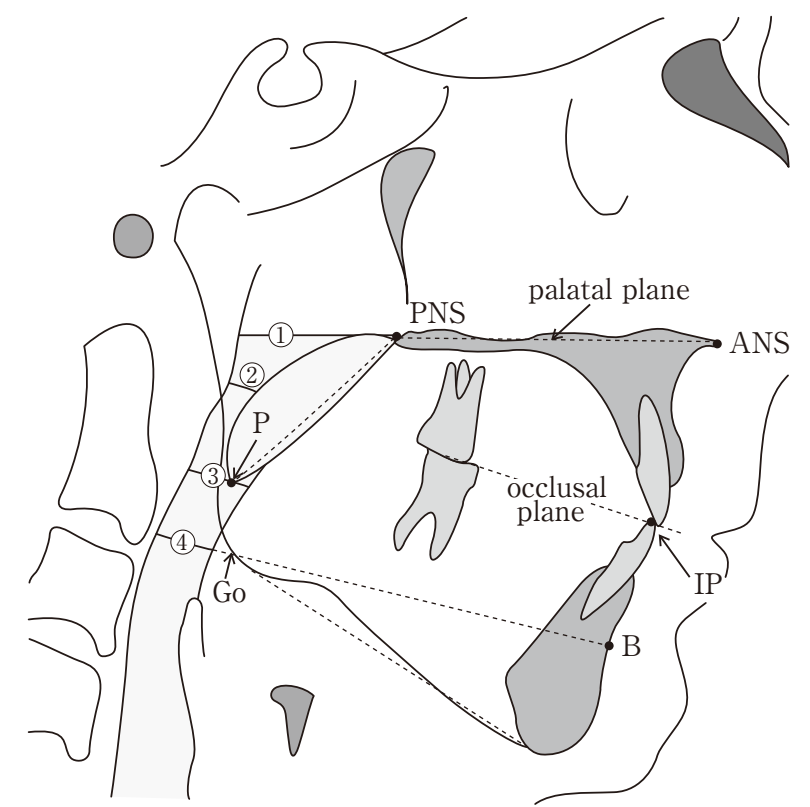

Fig. 1 Cephalometric landmarks and line measurements. ANS: Anterior nasal spine, PNS: Posterior nasal spine, Go: Gonion, IP: Incisal point, P: Tip of soft palate, B: Supramentale.

Liner measurements: (1) PNS-AS: Point nasal spine airway space. The thickness of an extension of the palatal plane to the posterior pharyngeal wall; (2) SPAS: Superior posterior airway space. The thickness of the airway behind the soft palatal along a line parallel to the Go-B line; (3) MAS: Middle airway space. The thickness of the airway along to parallel line to the Go-B line through P; (4) IAS: Inferior airway space. The thickness of the airway along a line extended through the Go-B line

The horizontal distances of the oropharyngeal space (PNS-AS, SPAS, MAS and IAS at Fig. 1) were measured with and without the OA, and in the maximum opening position. The landmarks and reference lines use are defined in Figure 1. The analysis of the anterior and downward displacements of the condyle in the maximum opening position and with the OA were evaluated as compared with the condyle position of intercuspal position, resulting in their conversion to an $\mathrm{X}-\mathrm{Y}$ coordinate system. All analyses were performed by the same researcher.

\section{Pulse oximetry}

Recording of $\mathrm{SpO}_{2}$ was performed twice, with and without the OA, by pulse oximetry with a finger probe (PULSOX ${ }^{\circledR}$-M24; TEIJIN Co., Tokyo, Japan). The oxygen desaturation index (ODI 4\%) was calculated for events with decreases in $\mathrm{SpO}_{2}$ $>4 \%$ and a cumulative percentage of time spent at saturations below $90 \%\left(\mathrm{CT}_{90}\right)$ during sleeping was calculated.

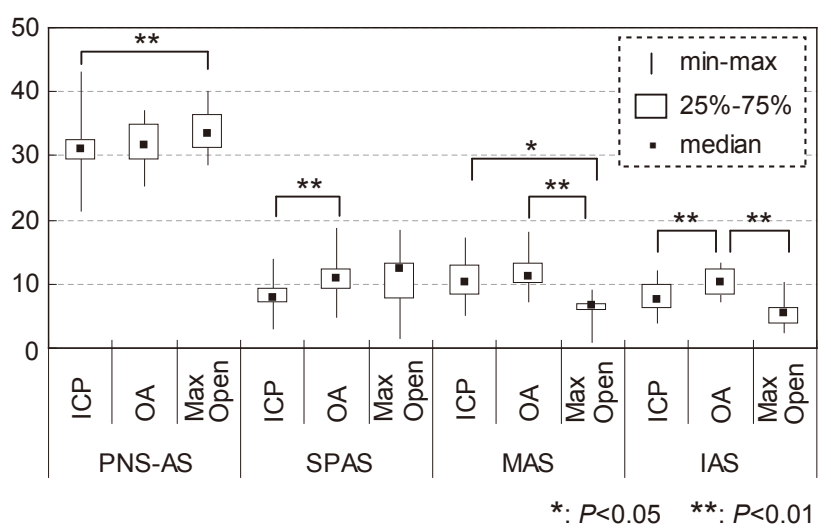

Fig. 2 The median values of HDOS in OSAS patients with and without the $\mathrm{OA}$ and at the maximum opening position.

\section{Statistical analysis}

The Friedman test was used to compare the horizontal distance of the oropharyngeal space (HDOS) both with and without the OA, and the maximum opening position. Spearman's rank correlation test was used to measure the correlation between the increase in HDOS and shift of the incisal point (IP), and condylar position with the OA. The ODI $4 \%, \mathrm{CT}_{90}$ and HDOS were compared for all subjects with and without the OA using the Wilcox ranking test. Values of $P<0.05$ indicated significance.

\section{Results}

\section{The values of HDOS in OSAS patients with and without the $\mathrm{OA}$ and at the maximum opening position}

The median values of HDOS in OSAS patients with and without the $\mathrm{OA}$ and at the maximum opening position are shown in Figure 2. There were significant differences among patients with and without the OA, and at the maximum opening position for each of the four HDOS measurements. Namely, the HDOS values for IAS and MAS showed significant decreases at the maximum opening position; however, for PNS-AS there was a significant increase at the maximum opening position. On the other hand, in the SPAS and IAS, HDOS tended to increase with the OA.

\section{Correlation between the variation of HDOS and the shift of IP, the condylar position with the $\mathbf{O A}$}

There was a relationship between the increase in HDOS and the anterior shift of IP with the 


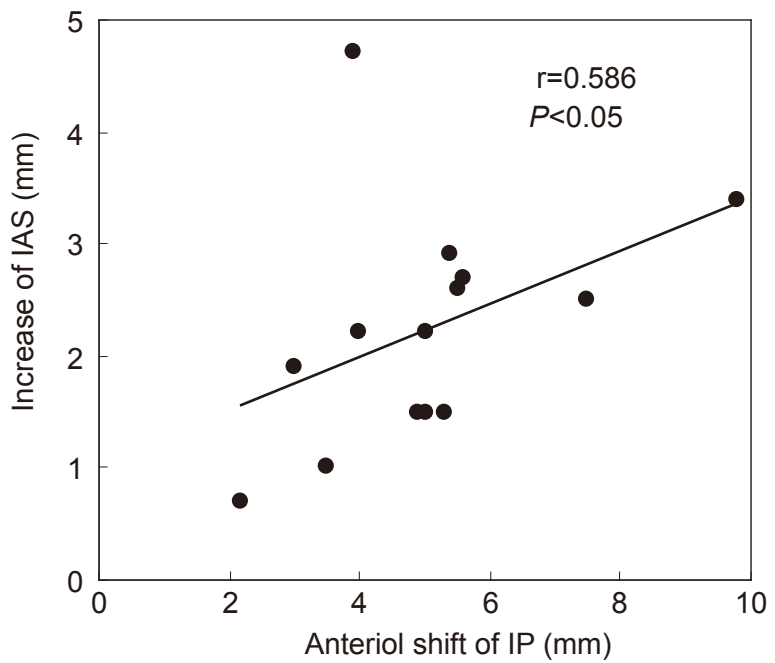

Fig. 3 Correlation between the increase in IAS and the anterior shift of incisal point with the OA.

$\mathrm{OA}(\mathrm{r}=0.586, P<0.05)$ (Fig. 3). However, there was no correlation between the variation in HDOS and the downward shift of IP with the OA. Similarly, there was a relationship between the increase in SPAS and the anterior shift of the condyle with the $\mathrm{OA}(\mathrm{r}=0.804, P<0.001)$ (Fig. 4). On the other hand, there was no relationship between the variation in HDOS and the downward shift of the condyle with the OA.

\section{Arterial oxygen saturation with and without the OA}

Values of ODI $4 \%$ and $\mathrm{CT}_{90}$ with and without the $\mathrm{OA}$ are shown in Figure 5. Values decreased significantly with the $\mathrm{OA}(P<0.01)$.

\section{Discussion}

It is noteworthy that an oral appliance was used by Robin $\mathrm{P},{ }^{18}$ to treat the glossoptosis in infants with micrognathia even in 1934 . The aim of using oral appliances is to widen the oropharyngeal airway by repositioning the mandible advancement during sleep. The American Academy of Sleep Medicine ${ }^{1}$ has issued practice guidelines stating that oral appliance therapy is indicated for simple snoring and mild OSAS and for moderate to severe OSAS if NCPAP is not acceptable or if surgery is inappropriate. Although NCPAP has been considered superior than oral appliances, it is poorly tolerated and less often used by many patients. Therefore, oral appliances provide an

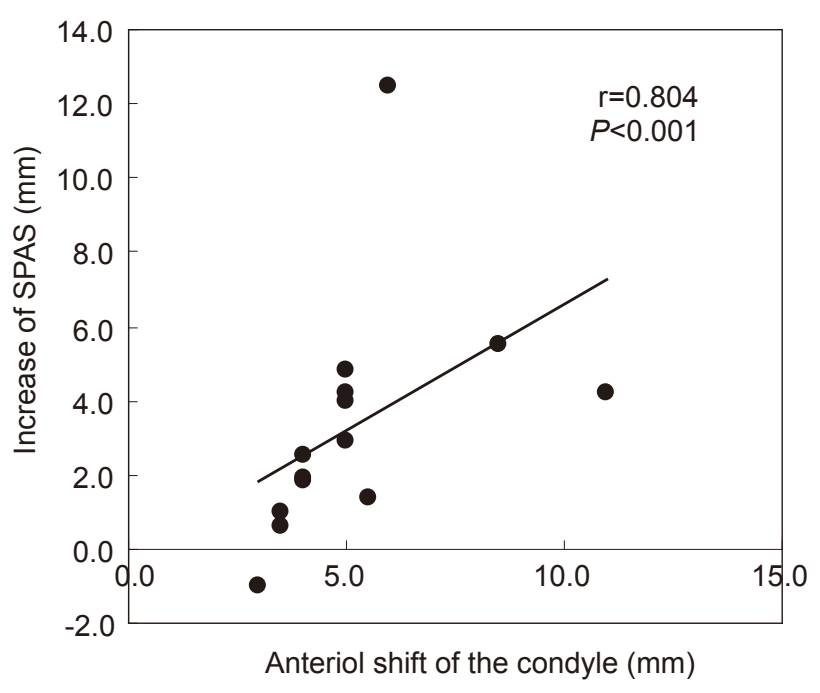

Fig. 4 Correlation between the increase in SPAS and the anterior shift of the condylar with the OA.

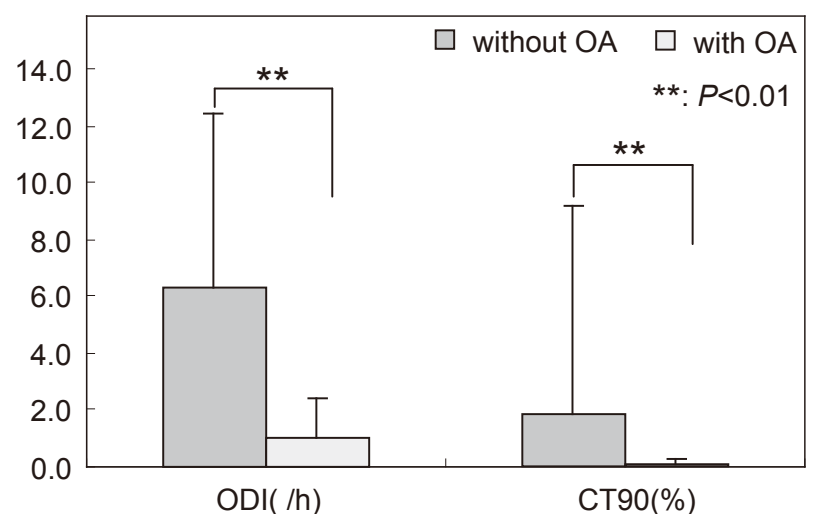

Fig. 5 Values of ODI $4 \%$ and $\mathrm{CT}_{90}$ with and without the OA.

important treatment for OSAS and may be the preferred initial treatment. At present, OAs have emerged as an increasingly popular alternative to more established therapies. ${ }^{19}$ However, it is possible that excessive mandibular protrusion could have detrimental consequences in the long term, especially for occlusion and the temporomandibular joint, and this requires further investigation.

In the present study we investigated the effects of using an OA in OSAS patients on arterial oxygen saturation and we assessed the relationships of OA use with variation in condylar position, the shift of IP, and HDOS.

Formal polysomnography is the gold standard for the diagnosis of OSAS, but the cost and inconvenience of polysomnography make alternative diagnostic methods desirable. Numerous rela- 
tively elaborate substitutes for polysomnography have been developed. Gyulay $\mathrm{S}$ et $\mathrm{al}^{20}$ concluded that home oximetry is suitable for screening for OSAS. Therefore, ODI $4 \%$ and $\mathrm{CT}_{90}$ could be considered an indication for hypoxia, during apnea.

Cephalometry has been used in a number of studies for assessing skeletal and soft tissue in OSAS patients in comparison with healthy persons. ${ }^{21,22}$ Yildrim $\mathrm{N}$ et $\mathrm{al}^{23}$ reported that uvular width in both OSAS patients and non-snoring, normal individuals increased in the supine position compared with the upright position. Fransson $\mathrm{AM}$ et $\mathrm{al}^{24}$ found that the use of a mandibular-protruding device increased pharyngeal space at all analyzed levels in both the upright and the supine positions. Thus, it may be assumed that a similar effect occurs in the hypopharyngeal area with the mandibular-protruding device. In this study, cephalometric radiographs were made with the OSAS patients in an upright position; however, this does not reflect the conditions during sleep. Therefore, it is recommended that the cephalograms be taken in the supine position.

The result of the presented study indicates that the values of ODI $4 \%$ and $\mathrm{CT}_{90}$ with the $\mathrm{OA}$ are lower than those values without the OA, which demonstrates the positive effect of the OA.

The translation of condyle position with the OAs was less than that observed in the maximal open position. At the maximum opening, the PNS-AS oropharyngeal space was greater without the OA; on the other hand, the MAS and IAS of oropharyngeal spaces were significantly lower with the OA. Structurally, the oropharyngeal airway is surrounded by soft tissue such as the tongue and soft plate, which is enclosed by body structures such as the mandible and vertebra. We consider that these findings can be explained by a mechanical model of the pharyngeal airway ('Meat and Container Balance' model ${ }^{25}$ ). This model indicates that an OA designed to advance the mandible forward increase the mandibular enclosure size, and therefore increases the container size, improving the balance. ${ }^{26}$

The top of the oropharyngeal space with the OA (i.e., PNS-AS) has not yet been confirmed with any positive results; however, there were significant increases in SPAS and IAS. The activity of the suprahyoid muscles appears to be improved by the protrusion of the mandible with the OA and that the horizontal distances of SPAS and IAS are also positively affected. Furthermore, there was a correlation between the increase in the horizontal distance of the IAS and the extent of the anterior shift of the incisor point, but no significant correlation with the downward shift of the incisor point. The above facts are consistent with our previous paper. ${ }^{15}$ In addition, we found that there was a significant correlation between the increase in the horizontal distance of SPAS and the anterior shift of the condyle. However, the correlation with the downward shift of the condyle was not significant. These results suggest that mandibular protrusion using an $\mathrm{OA}$ in patients with OSAS demonstrates the positive effect of an increase in oropharyngeal space, and that the increase in vertical dimension with the use of the OA has a negative effect in OSAS patients.

\section{Conclusion}

The present study suggests that increasing the anterior shift in mandibular position using an OA designed to advance the mandible forward is useful as a cure for OSAS patients, and that excessive increase in the vertical dimension in $\mathrm{pa}^{-}$ tients using an OA was ineffective in expanding the oropharyngeal space.

\section{References}

1. American Academy of Sleep Medicine: Sleep related breathing disorders in adults: Recommendations for syndrome definition and measurement techniques in clinical research. Sleep 22: 667-689, 1999.

2. Millman RP. Daytime hypertension in obstructive sleep apnea. Prevalence and contributing risk factors. Chest 99: 861-866, 1995.

3. Strohl KP, Bonnie RJ, Findley L et al. Sleep apnea, sleepiness, and driving risk. Am J Respir Crit Care Med 150: 1463-1473, 1994.

4. Sullivan CE, Berthon-Jones M, Issa FG et al. Reversal of obstructive sleep apnea by continuous airway pressure applied through the nares. Lancet 1: 862-865, 1981.

5. Schmidt-Nowara W, Low AA, Wiegand L et al. Oral appliances for the treatment of snoring and obstructive sleep apea: A review. Sleep 18: 501-510, 1995.

6. Nakazawa Y, Sakamoto T, Yasutake R et al. Treatment of sleep apnea with prosthetic mandibular advancement (PMA). Sleep 15: 499-504, 1992.

7. Yoshida K. Effects of a mandibular advancement device for the treatment of sleep apnea syndrome 
and snoring on respiratory function and sleep quality. J Craniomandibular Pract 18: 98-105, 2000.

8. Lowe AA, Sjöholm TT, Ryan CF et al. Treatment, airway and compliance effects of a titratable oral appliance. Sleep 23: S172-179, 2000.

9. Ryan CF, Love LL, Peat D et al. Mandibular advancement oral appliance therapy for obstructive sleep apnoea: effect on awake caliber of the velopharynx. Thorax 54: 972-977, 1999.

10. Lowe AA. Can we predict the success of dental appliance therapy for the treatment of obstructive sleep apnea based on anatomic considerations? Sleep 16: S93-95, 1993.

11. Gao XM, Zeng XL, Fu MK et al. Magnetic resonance imaging of the upper airway in obstructive sleep apnea before and after oral appliance therapy. Chin J Dent Res 2: 27-35, 1999.

12. Schmidt-Nowara WW, Meade TE, Hays MB. Treatment of snoring and obstructive sleep apnea with a dental orthosis. Chest 99: 1378-1385, 1991.

13. Tsuiki S, Hiyama S, Ono T et al. Effects of a titratable 0ral appliance on supine airway size. Sleep 24: 554-560, 2001.

14. Tsuiki S, Ono T, Kuroda T. Mandibular advancement modulates respiratory-related genioglossus electromyographic activity. Sleep Breath 4: 53-57, 2000.

15. Ferguson KA, Lowe AA. Oral appliances for sleepdisordered breathing. In: Krygen MH, Roth T, Dement WC eds. Principles and practice of sleep medicine $4^{\text {th }}$ edition: 1098-1108 Philadelphia: WB Saunders, 1098-1108, 2005.

16. Lui Y, Lowe AA, Fleerham JA et al. Cephalometric and physiologic predictors of the efficacy of an adjustable oral appliance for treating obstructive sleep apnea. Am J Orthod Dentofacial Orthop 120: 639-645, 2001.

17. Harada M, Inoko Y, Shimizu K et al. The effectively of prosthodontics mandibular advance- ment for obstructive sleep apnea syndrome. J Jpn Prosthoudont Soc 48: 733-740, 2004.

18. Robin P. Glossoptosis due to artesia and hypotrophy of the mandible. Am J Dis Child 48: 541-547, 1934.

19. Standard of Practice Committee of the American Sleep Disorders Association: Practice parameter for the treatment of snoring and obstructive sleep apnea with oral appliance. Sleep 18: 511-513, 1995.

20. Gyulay S, Olson LG, Hensley MJ et al. A comparison of clinical assessment and home oximetry in the diagnosis of obstructive sleep apnea. Am Rev Respir Dis 147: 50-53. 1993.

21. Battagel J, Johal A, Kotecha B et al. A cephalometric comparison of subjects with snoring and obstructive sleep apnoea. Eur J Orthod 22: 353-365, 2000.

22. Hoekenma A, Hovinga B, Stegenga B et al. Craniofacial morphology and obstructive sleep apnoea: a cephalometric analysis. J Oral Rehabil 30: 690-696, 2003.

23. Yildrim N, Fitzpatrick F, Whyte KF et al. The effect of posture on upper airway dimensions in normal subjects and in patients with sleep apnea/hypopnea syndrome. Am Rev Respir Dis 144: 845-847, 1991.

24. Fransson AM, Svenson BA, Isacsson G. The effect of posture and mandibular protruding device on pharyngeal dimensions: a cephalometric study. Sleep Breath 6: 55-68, 2002.

25. Isono S. Contribution of obesity and craniofacial abnormalities to pharyngeal collapsibility in patients with obstructive sleep apnea. Sleep \& Biol Rhythm 2: 17-21, 2004.

26. Kato J, Isono S, Tanaka A et al. Dose-dependant effects of mandibular advancement on pharyngeal mechanics and nocturnal oxygenation in patients with sleep-disordered breathing. Chest 117: 1065-1072, 2000. 